\title{
Serious video games and virtual reality for prevention and neurorehabilitation of cognitive decline because of aging and neurodegeneration
}

\author{
Arseny A. Sokolov ${ }^{\mathrm{a}, \mathrm{b}, \mathrm{c}}$, Amélie Collignon ${ }^{\mathrm{a}, \mathrm{d}}$, \\ and Mélanie Bieler-Aeschlimann ${ }^{\mathrm{e}, \mathrm{f}}$
}

\begin{abstract}
Purpose of review
Cognitive decline because of aging and neurodegeneration has become increasingly prevalent. This calls for the implementation of efficacious, motivating, standardized and widely available cognitive interventions for the elderly. In this context, serious video games and virtual reality may represent promising approaches. Here, we review recent research on their potential for cognitive prevention and neurorehabilitation of agerelated cognitive decline and mild cognitive impairment (MCI).
\end{abstract}

\section{Recent findings}

The majority of currently available data in this evolving domain lacks the methodological quality to draw reliable conclusions on the potential of novel technology for cognitive training in older people. However, single well designed randomized controlled trials have reported promising effects of cognitive interventions involving serious video games and virtual reality. The cognitive benefits of exergames promoting physical exercise with and without combined cognitive training remain unclear.

\section{Summary \\ The immersion into stimulating and motivating environments along with training content based on neuroscientific and neuropsychological models may represent a significant advance as compared with conventional computerized cognitive training. Additional research with sound methodology including sufficient sample sizes, active control groups and meaningful outcome measures of everyday function is needed to elucidate the potential of serious video games and virtual reality in multifactorial neurorehabilitation of cognitive decline in aging and neurodegeneration.}

\section{Keywords}

aging, cognitive neurorehabilitation, computerized cognitive training, exergames, neurotechnology, serious video games, virtual reality

\section{INTRODUCTION}

As the proportion of older individuals increases in the general population and neurological practice, we will face cognitive decline more frequently. Among other challenges, this calls for efficacious interventions for prevention and neurorehabilitation of cognitive decline in the elderly. According to some large-scale studies, conventional neuropsychological interventions may not only improve the targeted domains but also benefit everyday life function in healthy older adults (HOA) and people with mild cognitive impairment (MCI). For instance, the Advanced Cognitive Training for Independent and Vital Elderly (ACTIVE) randomized controlled trial (RCT) in 2832 HOA reported domain-specific effects of 10 sessions of inductive reasoning versus processing speed versus verbal

\begin{abstract}
aNeuroscape@NeuroTech Platform \& Service de Neurologie, Département des Neurosciences Cliniques, Centre Hospitalier Universitaire Vaudois (CHUV), Lausanne, ' Department of Neurology, University Neurorehabilitation, University Hospital Inselspital, University of Bern, Bern, Switzerland, "Neuroscape Center, Weill Institute of Neuroscience, Department of Neurology, University of California, San Francisco, USA, ${ }^{\mathrm{d} C l i n i c a l}$ and Experimental Neuropsychology Laboratory, Department of Psychology, University of Geneva, Geneva, 'Leenaards Memory Centre, Département des Neurosciences Cliniques, Centre Hospitalier Universitaire Vaudois (CHUV) and ${ }^{\dagger}$ MindMaze SA, Lausanne, Switzerland

Correspondence to Arseny A. Sokolov, MD, Neuroscape@NeuroTech Platform \& Service de Neurologie, Département des Neurosciences Cliniques, Centre Hospitalier Universitaire Vaudois (CHUV), Rue du Bugnon 46, CH-1011 Lausanne, Switzerland. Tel: +41 795567 355; e-mail: arseny.sokolov@chuv.ch
\end{abstract}

Curr Opin Neurol 2020, 33:239-248

DOI:10.1097/WCO.0000000000000791 


\section{KEY POINTS}

- Serious video games and virtual reality are promising approaches for cognitive training and neurorehabilitation in older people.

- This novel technology can increase motivation and training effects through immersion in stimulating environments.

- Closed-loop adaptivity of difficulty in real-time may represent a major advance in cognitive training.

- The currently available data do not allow drawing reliable conclusions on the efficacy and assumed advantages of serious video games and virtual reality.

- Large-scale RCTs with state-of-the-art methodology are required to elucidate the potential of this neurotechnology for cognitive training and neurorehabilitation of cognitive decline related to aging or neurodegeneration.

episodic memory training $[1,2]$. Although no selfreported instrumental activities of daily living (IADL) benefits were observed in either group at 2 years after training [1], inductive reasoning training was associated to IADL improvements at 5-year follow-up [3]. Somewhat surprisingly, such distal transfer from specific cognitive training to everyday life function appeared in all three interventional groups at the ten-year follow-up [4]. A recent RCT in 145 individuals with MCI comparing $16 \mathrm{~h}$ of training on strategies for memory and attentional control (MEMO+) to a psychosocial training and a no-contact control group reported specific improvements in memory performance on cognitive testing and in everyday mnemonic strategies as assessed by the Multifactorial Memory Questionnaire in the $\mathrm{MEMO}+$ group [5"']. The effects persisted at follow-up assessments after 3 and 6 months.

Distal transfer and long-term persistence of benefits constitute the ultimate objectives of cognitive interventions, but have been rarely observed [6]. Proximal and transient transfer, from the trained cognitive function to a related cognitive domain, has been reported more frequently. Furthermore, the majority of current clinical and experimental procedures aiming at prevention or deceleration of cognitive decline because of aging and neurodegeneration have only yielded limited benefits $[7,8]$. In the era of information and communication technology, a part of the scientific community has started developing digital solutions to extend standard neuropsychological approaches for cognitive training and overcome the main limitations of currently available cognitive interventions for $\mathrm{HOA}$ and people with MCI. Interventions encompassing novel technology have become widely accepted in cognitive neurorehabilitation [9]. Here, we review recent research in this area, with a particular focus on the past 2 years and the use of neurotechnology, such as serious video games and virtual reality.

\section{COMPUTERIZED COGNITIVE TRAINING}

Computerized cognitive training (CCT) usually represents translation and digitalization of standard neuropsychological interventions in a computerized framework. Digitalization offers several advantages, such as a high level of standardization and reproducibility, time efficiency and the possibility for remote training. Of note, already the ACTIVE trial discussed above employed CCT for processing speed training [2]. Furthermore, an RCT involving $30 \mathrm{HOA}$ and 30 younger adults indicated that $8 \mathrm{~h}$ of computerized divided attention training improved specifically attentional control capacities and dualtask performance in HOA as opposed to sequential training on the same tasks that required focused attention [10"']. Transfer was reported with respect to dual-tasking in an ecological virtual reality scenario, but no effects were found on the self-reported Cognitive Failures Questionnaire. Overall, systematic literature reviews on CCT in HOA and patients with dementia have suggested significant albeit modest positive effects $[11,12]$. However, the available evidence indicates only limited transfer in HOA and is insufficient with respect to benefits of CCT in MCI [13]. Apart from the processes underlying cognitive decline and potentially limiting the effects of training irrespective of the modality, these modest benefits could be because of a relative lack of motivation and personalization in conventional neuropsychological interventions, irrespective of whether digital tools are used.

\section{FROM COMMERCIAL TO SERIOUS VIDEO GAMES}

The fun and joy of video games could enhance the inherent motivation for training in people with cognitive decline [14]. An RCT in 36 HOA found that, when compared with a no-contact control group, playing the action video game Super Mario Bros three times a week over 2 months yielded more wide-spread cognitive improvement (with a particular emphasis on the visuospatial and working memory domains) than the same training dose of the reasoning-oriented, off-label Dr Kawashima's Brain Training [15"]. Although these data indicate that the rich environment and broad challenges of commercial action video games 
without cognitive specificity may afford a more global improvement in cognitive function, the only significant interaction across all three conditions favored Dr Kawashima's Brain Training with respect to specific improvement in the Stroop test. In a similar vein, an RCT in $54 \mathrm{HOA}$ showed that $60 \mathrm{~h}$ of an off-label, gamified visual attention and processing speed CCT (PositScience InSight) outperformed the commercial action video game Crazy Taxi and a no-contact control group [16"] in the Useful Field of View (UFOV) assessment of processing speed, selective and divided attention [17]. No significant differences between the groups were found on IADL.

These outcomes may reflect the controversy in defining useful and meaningful outcome measures in research on cognitive interventions. Yet, it may also suggest that a lack of cognitive specificity and neuroscience-informed design limits the utility of commercial video games for cognitive neurorehabilitation. Furthermore, commercial action video games have been typically designed for younger individuals, and may not be well accepted and tolerated by older people [18]. In contrast, commercially available, computerized puzzle games are enjoyed by HOA [19].

Combining the specific elements of CCT and the motivational aspects of commercial video games has led to the development of serious video games $[20,21]$. Through integration of neuroscientific models, neuropsychological content with immersive graphics and soundtrack, serious video games may bear a significant potential for cognitive neurorehabilitation across several neurological and psychiatric conditions [20,22,23]. Furthermore, serious video games can be designed to involve monitoring of multiple measures, such as reaction time, response accuracy, precise duration of practice, as well as physiological indicators like heart rate, skin conductance, eye movements or brain activity. These measures can be used for real-time feedback, detailed recording of training performance and progression, as well as closed-loop adaptation [24]. Closed-loop adaptive video games (CLAVs) incorporate real-time, performance-driven adaptation of game challenges [20]. A landmark study in 46 HOA showed that a custom-designed dual-task CLAV improved significantly divided attention as opposed to single-task and no-contact control conditions [25]. The data also indicated transfer to sustained attention and working memory that were not targeted directly. Furthermore, training benefits persisted at 6 months follow-up. Good acceptance of serious video games has been reported among individuals with neurodegenerative disease [26].

\section{EXERGAMES}

Physical exercise alone has been shown to yield cognitive effects in older people $[27,28]$. However, the adherence to physical exercise is difficult to maintain. Exergames are defined as video games aiming primarily at physical training [29]. Although exergames do not appear to outperform conventional physical training in terms of cognitive effects [30], they may be a motivating vector to promote adherence to physical exercise. Recent meta-analyses and systematic reviews indicated that playing exergames can benefit executive function, attention and visuospatial processing in $\mathrm{HOA}$ and $\mathrm{MCI}$ $[30,31]$, although the results of the former have been challenged [32]. In an RCT in 78 individuals with MCI, playing sports video games on the Nintendo Wii for $30 \mathrm{~min}$ three times a week over 10 weeks yielded more significant effects in the digit span (working memory) and also health-related quality of life than the same dose of the CoTras CCT program [33"]. Conversely, no differential effects were found in verbal learning, short-term verbal memory or long-term visual memory.

As the combination of physical and cognitive training appears to outperform physical or cognitive exercise alone in terms of cognitive benefits [34,35], coupling exergames with cognitive training may be a promising avenue. In this respect, a recent promising RCT in 44 individuals with MCI evaluated the cognitive and electrophysiological effects of the off-label Dr Kawashima's Body and Brain Exercises on the Microsoft Xbox 360 Kinect that allows tracking movement in response to cognitive tasks displayed on a large screen [36"']. The control group performed nondigital motion range exercises without cognitive content and the training dose in each group was 25$30 \mathrm{~min}, 5$ days a week during 6 weeks $(12.5-15 \mathrm{~h})$. As compared with the control, the cognitive exergame afforded significant benefits on the Mini-Mental State Examination and Montreal Cognitive Assessment scores, as well as the Trail Making Test B assessing cognitive flexibility. Some electrophysiological effects were also described. However, these encouraging results may have been somewhat confounded by probable differences in the expectancy of cognitive benefits between the two training groups [37]. Between-group differences other than the training content, such as training location or the presence of a therapist can further increase the divergence in expectancy, and thus training outcomes.

The Aerobic and Cognitive Exercise Study (ACES) enrolled 111 older participants (including 83 individuals with suspected MCI) to evaluate the effects of stationary cycling exercise coupled with a serious video game versus cycling in a virtual landscape (with 45 and 46 participants, respectively). 
Both the landscape and video game were displayed on a screen mounted on the stationary bike. A game-only condition without physical exercise was also conducted, with five randomized and ten attributed participants. However, no participant completed the 6-month training in this control group. In the other two conditions, only seven participants per principal condition completed 6 months of training, representing an attrition of $87 \%$. The study comprised pretraining and posttraining structural MRI, saliva exosome analyses, as well as plasma protein assays for brain-derived neurotrophic factor, C-reactive protein, insulin-like growth factor 1, interleukin 6 and vascular endothelial growth factor. No differences between the more passive and the cognitively demanding exergame were found on the primary cognitive outcome measures [38]. However, the exer-tour cycling without elevated cognitive demands afforded significantly greater benefits on the secondary outcomes verbal memory, physical fitness and everyday life cognition. The greater effects for passive cycling as opposed to exergaming contradicted the results of a preceding ACES study [39]. The same research group also evaluated the offlabel, home-based interactive Physical and Cognitive exercise system (iPACes) that involved pedaling on an elliptical and completing a list of errands along a virtual bike path displayed on a tabletlaptop. In the absence of a control group, 10 out of 31 enrolled older individuals completed a twiceweekly training over 3 months and exhibited significant improvement in the Stroop test and Alzheimer's Disease Assessment scale delayed word recall that measure inhibition control and verbal memory, respectively [40]. Eight participants with MCI or mild dementia completed another pilot study comparing stationary cycling coupled with cognitive challenges (such as avoiding cars or shopping in a supermarket) to a no-contact control group. The trial did not find significant differences in cognitive outcomes [41]. The question of whether the inclusion of cognitive training in an exergame context may be useful for maintaining or rehabilitating cognitive function, thus remains largely open.

\section{VIRTUAL REALITY FOR TRAINING AND ASSESSMENT}

Virtual reality may represent another important neurotechnology for optimization of cognitive training. This technology allows immersion and interaction in virtual environments. The use and manipulation of such environments may open novel perspectives for cognitive neurorehabilitation [42-44]. Although cognitive training on a desktop screen has also been described as virtual reality, because of recent technological and conceptual progress, only immersion in a virtual environment via a room-sized cube (CAVE system) or head-mounted displays should be referred to as virtual reality.

Of particular significance for older adults, virtual reality allows personalized and ecological assessment and training of IADL. In a study across 25 HOA and individuals with MCI without a control condition, nonimmersive computerized IADL training was reported to improve visual memory, attention and cognitive flexibility, but without affecting everyday life cognition [45]. Recent research in 42 older individuals with MCI concluded that IADL training and physical exercise in and outside virtual reality yield largely similar outcomes, with the immersive virtual reality group showing more benefits on the Trail Making Test B (cognitive flexibility) and gait cadence during cognitive-motor dual tasking [46"].

Exposure to and immersive interaction with virtual environments may also be useful for assessing and training spatial, episodic and prospective memory [47-49]. Furthermore, patients with MCI and dementia have been reported to prefer immersive virtual reality to paper-and-pencil interventions [50]. This was particularly the case for participants suffering from apathy, further underlining the motivational value of novel technology. Additional, carefully designed RCTs are needed to assess the true potential of immersive virtual reality for cognitive neurorehabilitation in HOA and people with MCI.

\section{TOWARDS MULTIMODAL NEUROTECHNOLOGICAL AND MULTIFACTORIAL INTERVENTIONS}

The potential of more holistic, multifactorial interventions addressing several functional domains to improve physical and cognitive health has become increasingly recognized [51]. The 2-year longitudinal Finnish Geriatric Intervention Study to Prevent Cognitive Impairment and Disability (FINGER) study indicated that a multifactorial intervention involving diet, exercise, cognitive training and vascular risk monitoring could improve or maintain cognitive function in 1260 older people at-risk of cognitive decline [52]. The currently ongoing ENGAGE RCT represents an interesting attempt at integrating cognitive training with novel technology and leisure activities [53]. One hundred and forty-four older adults with subjective memory deficits will be assigned to either the ENGAGE-MUSIC/SPANISH interventional or the ENGAGE-DISCOVERY active control condition for a total of $48 \mathrm{~h}$ over 4 months. ENGAGE-MUSIC/SPANISH consists of learning 


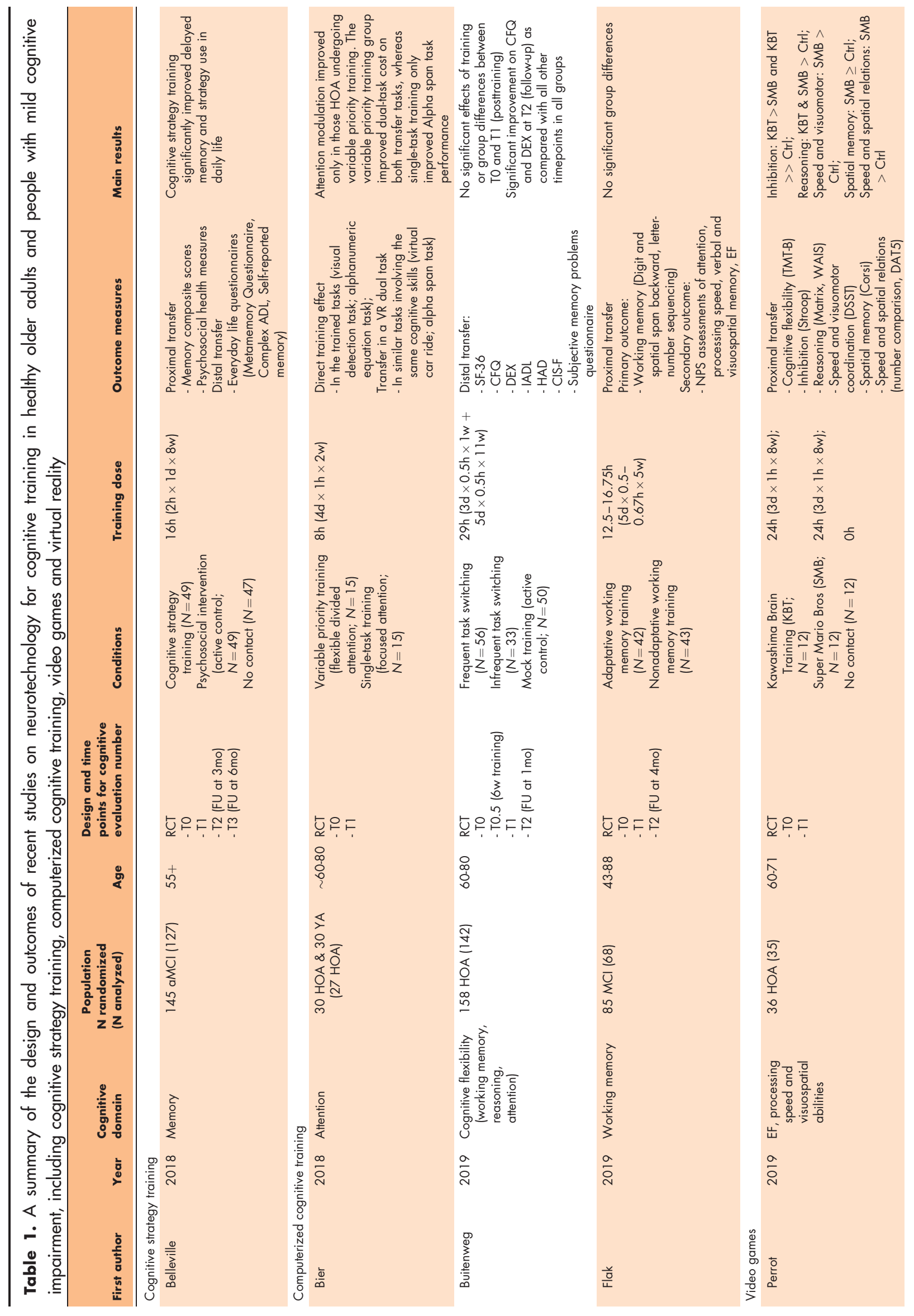




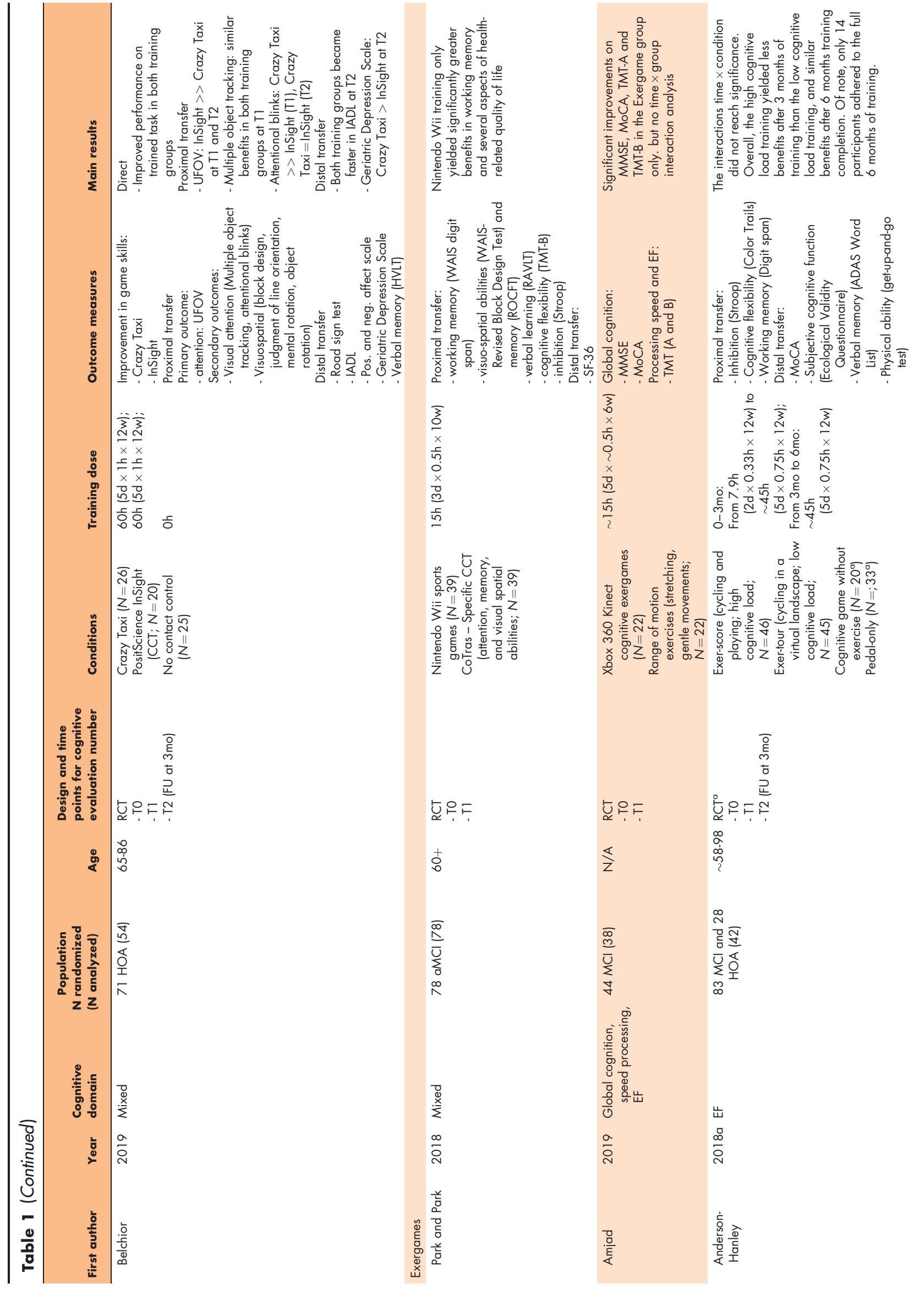




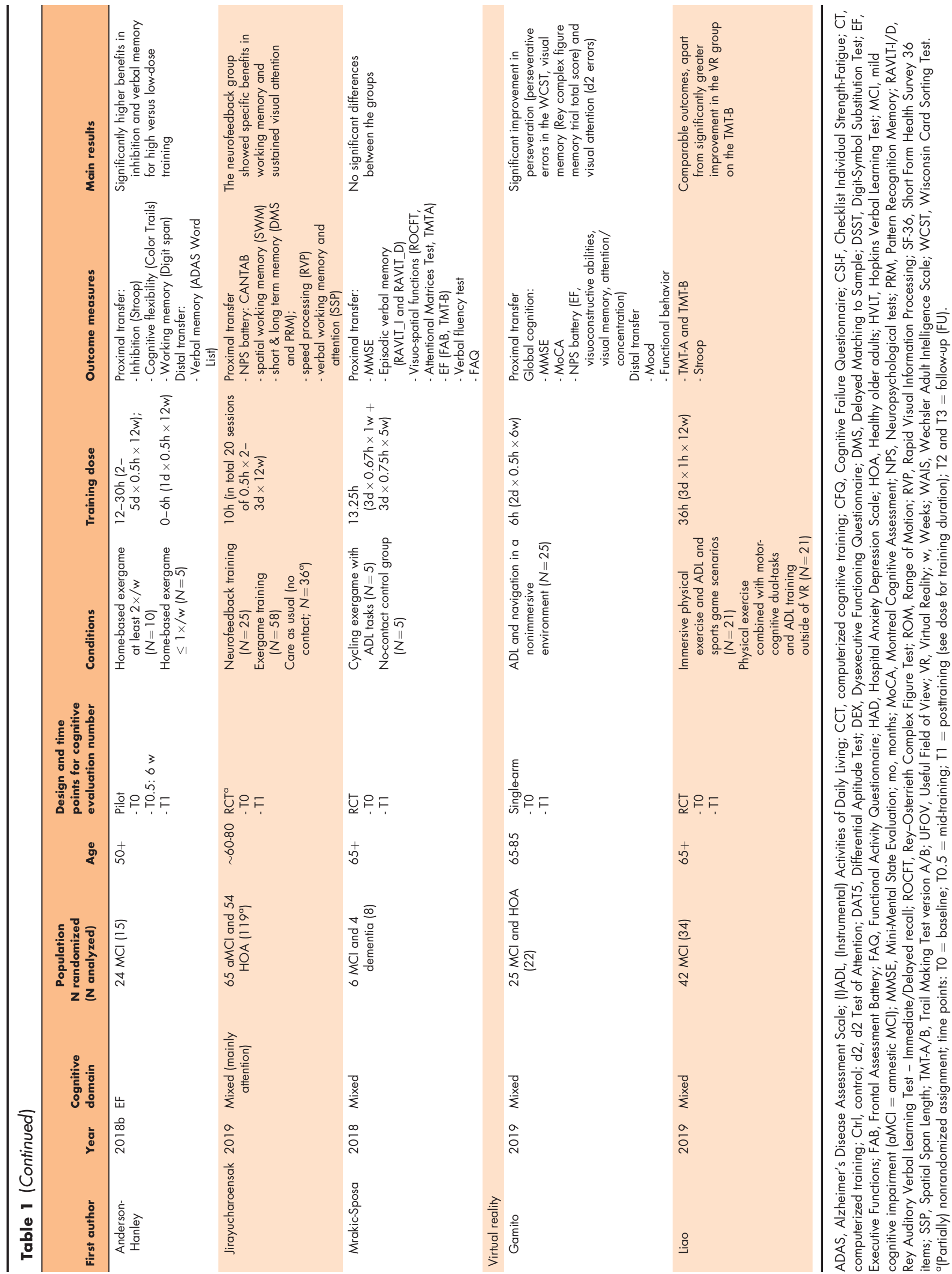


attentional control and memory strategies, applying this knowledge to a leisure activity (learning music or Spanish as a second language), and playing commercially available video games targeting attention. ENGAGE-DISCOVERY involves psychoeducation (on cognition and the brain), cultural and social interactions (documentary viewing with discussions) and playing commercial video games with a low cognitive load. The primary outcome is an episodic memory composite score. Secondary outcome measures focus on attentional control. Furthermore, the study will assess effects on psychological health and daily life as well as structural and functional brain plasticity. In addition, evaluation of potential covariates such as cognitive reserve (education and lifestyle), sex and genotype (apolipoprotein E4, brainderived neurotrophic factor and catechol-O-methyltransferase) will be used towards differentiated data interpretation. Multifactorial interventions targeting not only specific cognitive functions and emotional processing but also meta-cognitive abilities, as well as physical and social activities appear more promising than single-domain training.

Other neurotechnological approaches should also be considered as alternatives or additional elements in multifactorial interventions. For instance, positive cognitive effects of a neurofeedback braincomputer interface approach were shown in a recent study [54"]. One hundred and nineteen HOA and people with MCI were assigned to three conditions: $10 \mathrm{~h}$ of neurofeedback + standard care, $10 \mathrm{~h}$ of exergame + standard care and standard care alone. In the neurofeedback condition, participants were trained to control a set of desktop video games by optimizing their sustained attention-related power ratio of beta to alpha frequency bands recorded by electroencephalography. The exergame condition consisted of five serious video games combining physical and cognitive challenges. The games were displayed on a giant screen and controlled by optical whole-body movement tracking. Both HOA and MCI participants in the neurofeedback condition exhibited significant improvements in sustained attention and spatial working memory strategy as compared with the exergame and standard care conditions. Conversely, individuals in the cognitive exergame condition showed greater improvements in visuospatial working memory (spatial span length).

\section{CONCLUSION AND OUTLOOK}

Taken together, the domain of serious video games and virtual reality for cognitive training and neurorehabilitation has just started to evolve. Single well designed and sufficiently powered RCTs over the past 2 years shed light on the potential of CCT, serious video games with and without coupled physical exercise and virtual reality for improving cognition in older people (Table 1). However, so far, the majority of research published in this realm lacks the methodological quality to afford reliable conclusions on the efficacy of this technology.

Future research using state-of-the-art methodology will help evaluate whether serious video games and virtual reality can be efficacious in preventing cognitive decline related to normal and pathological aging. Such RCTs should employ approaches that are easily accessible and accepted by older people $[22,26]$, as adherence may represent a significant issue $[38,40]$. The intervention design should take into account the specific needs of the elderly population [18]. This would not only ensure sufficient sample sizes for data interpretation but also indicate feasibility of the interventions in real life.

Irrespective of the vector, the training content is a key factor. Mere computerization of cognitive training appears insufficient for harnessing the considerable opportunities afforded by information and communication technology [6]. In addition to the highly engaging contexts, serious video games and particularly immersive virtual reality may transport the training participants outside of the clinical or interventional environment, and thus, further promote motivation, adherence and performance. Virtual reality may become a cost-efficient and mobile tool for highly ecological yet standardized cognitive training [22,44,46",55] and evaluation [55-57]. Most important, virtual reality allows environmental manipulations that would be impossible otherwise or require substantial efforts and resources [44].

Adaptivity is considered a key advantage of serious video games, although CCT can also be endowed with adaptivity. Closed-loop real-time adaptivity is believed to maintain the participant in an optimal range of effort, and thus, promote training effects [20]. The nature and time scale of adaptivity may play an important role. Level-wise adaptivity to constantly maximal effort did not enhance cognitive training effects when compared with similar nonadaptive CCT or video games $[58,59]$. Instant adaptation of training with both increases and reductions in difficulty based on performance [25] or correlates of brain activity [24] may, therefore, be more useful.

Furthermore, the choice of an appropriate active control condition appears indispensable. The absence of control groups or a no-contact control group do not allow for sufficiently meaningful conclusions. The active control should match the expectancy of the intervention without targeting the same cognitive mechanisms $[37,60]$. Defining useful primary and secondary outcome measures 
that assess not only specific training effects but also transfer to related cognitive domains (proximal transfer) and everyday life function (distal transfer) represents another significant challenge [61]. Ultimately, an important follow-up goal related to reallife and clinical use of interventions yielding promising initial effects will be to determine the optimal training intensity and dose.

Most important, among others, the studies reviewed here highlighted the compensatory potential of both HOA and individuals with MCI. One may argue that the underlying processes diminish the utility and, in particular, long-term benefits of cognitive interventions in the elderly. However, similar to younger patients with neurological conditions $[62,63]$, lower cognitive ability appears to predispose to greater cognitive benefits after training in older people $[45,64]$. This may also illustrate the need for personalized interventions tailored to the individual's specific needs and deficits. This personalization or stratification may also involve information beyond the cognitive profile, such as brain volumetry [65] or multimodal analyses of brain connectivity and its relationship to behavior [66]. If pharmacological options halting or decelerating neurodegeneration become available, cognitive interventions in MCI may eventually evolve into neurorehabilitation aiming at recovery.

In a nutshell, serious video games and virtual reality may complement existing neuropsychological interventions by affording a more engaging, standardized yet personalized context for cognitive training and neurorehabilitation. However, additional research with well designed content and control conditions taking into account the specific needs and expectations of the elderly and using state-ofthe-art methodology is required to elucidate the true potential of this neurotechnology. Given previous evidence, it appears unlikely that serious video games or virtual reality approaches will become standalone, highly efficacious approaches for age-related cognitive decline or MCI. They will rather complement multifactorial interventions involving physical exercise, diet, lifestyle adaptation, cognitive training and learning meta-cognitive strategies for coping and compensation. Neurotechnological approaches could represent a decisive component in multifactorial interventions for prevention and neurorehabilitation of cognitive decline related to normal aging and neurodegeneration.

\section{Acknowledgements}

The authors wish to thank Petr Grivaz and Alexis Renaud for technical assistance in preparation of this manuscript.

\section{Financial support and sponsorship}

The preparation of this article was supported by fellowships from the Leenaards Foundation, Swiss National Science Foundation, Synapsis Foundation - Alzheimer Research Switzerland ARS, SICPA Foundation and grants from the Helmut Horten Foundation and the Fondation Anna and André Livio Glauser to AAS. The contribution of MBA was supported by salary from MindMaze SA and a grant received from the Active and Assisted Living Programme, a European instance devoted to promoting technological solutions to elderly people.

\section{Conflicts of interest}

M.B.A. has been an employee of MindMaze SA, Lausanne, Switzerland.

\section{REFERENCES AND RECOMMENDED}

\section{READING}

Papers of particular interest, published within the annual period of review, have been highlighted as:

- of special interest

m. of outstanding interest

1. Ball K, Berch DB, Helmers KF, et al., Advanced Cognitive Training for Independent and Vital Elderly Study Group. Effects of cognitive training interventions with older adults: a randomized controlled trial. JAMA 2002; 288:2271-2281.

2. Jobe JB, Smith DM, Ball K, et al. ACTIVE: a cognitive intervention trial to promote independence in older adults. Control Clin Trials 2001; 22:453-479.

3. Willis SL, Tennstedt SL, Marsiske M, et al., ACTIVE Study Group. Long-term effects of cognitive training on everyday functional outcomes in older adults. JAMA 2006; 296:2805-2814.

4. Rebok GW, Ball K, Guey LT, et al., ACTIVE Study Group. Ten-year effects of the advanced cognitive training for independent and vital elderly cognitive training trial on cognition and everyday functioning in older adults. J Am Geriatr Soc 2014; 62:16-24.

5. Belleville S, Hudon C, Bier N, et al. MEMO+: efficacy, durability and effect of

-n cognitive training and psychosocial intervention in individuals with mild cognitive impairment. J Am Geriatr Soc 2018; 66:655-663.

This study illustrated the positive effects of mnemonic strategy learning on memory performance and everyday mnemonic strategies in individuals with $\mathrm{MCl}$, as well as durability of the benefits.

6. Simons DJ, Boot WR, Charness N, et al. Do 'brain-training' programs work? Psychol Sci Public Interest 2016; 17:103-186.

7. Bahar-Fuchs A, Martyr A, Goh AM, et al. Cognitive training for people with mild to moderate dementia. Cochrane Database Syst Rev 2019; 3:CD013069.

8. Martin M, Clare L, Altgassen AM, et al. Cognition-based interventions for healthy older people and people with mild cognitive impairment. Cochrane Database Syst Rev 2011; CD006220.

9. Cicerone KD, Goldin Y, Ganci K, et al. Evidence-based cognitive rehabilitation: systematic review of the literature from 2009 through 2014. Arch Phys Med Rehabil 2019; 100:1515-1533.

10. Bier B, Ouellet E, Belleville S. Computerized attentional training and transfer

n. with virtual reality: effect of age and training type. Neuropsychology 2018; 32:597-614.

This study introduced the first ecological training outcome measure in immersive virtual reality, and confirmed that variable dual-task training improves attentional control and ecological dual-task abilities.

11. Kueider AM, Parisi JM, Gross AL, Rebok GW. Computerized cognitive training with older adults: a systematic review. PLoS One 2012; 7:e40588.

12. Garcia-Casal JA, Loizeau A, Csipke $E$, et al. Computer-based cognitive interventions for people living with dementia: a systematic literature review and meta-analysis. Aging Ment Health 2017; 21:454-467.

13. Butler M, McCreedy $E$, Nelson VA, et al. Does cognitive training prevent cognitive decline?: a systematic review. Ann Intern Med 2018; 168:63-68.

14. Bavelier D, Green CS, Pouget A, Schrater P. Brain plasticity through the life span: learning to learn and action video games. Annu Rev Neurosci 2012;35:391-416.

15. Perrot $A$, Maillot $P$, Hartley $A$. Cognitive training game versus action videogame: - effects on cognitive functions in older adults. Games Health J 2019; 8:35-40. This work suggested more global cognitive benefits of a commercial action video game as opposed to a reasoning-oriented cognitive training video game, but only found significant interaction effects on cognitive flexibility for the latter. 
16. Belchior $P, Y a m A$, Thomas $K R$, et al. Computer and videogame interventions - for older adults' cognitive and everyday functioning. Games Health J 2019; 8:129-143.

These data indicated that gamified computerized cognitive training may yield more specific effects than commercial action video games.

17. Ball K, Owsley C. The useful field of view test: a new technique for evaluating age-related declines in visual function. J Am Optom Assoc 1993; 64:71-79.

18. Bediou $B$, Adams DM, Mayer RE, et al. Meta-analysis of action video game impact on perceptual, attentional, and cognitive skills. Psychol Bull 2018; 144:77-110.

19. Chesham A, Wyss $P$, Muri RM, et al. What older people like to play: genre preferences and acceptance of casual games. JMIR Serious Games 2017; 5:e8.

20. Mishra J, Anguera JA, Gazzaley A. Video games for neuro-cognitive optimization. Neuron 2016; 90:214-218.

21. Robert $\mathrm{PH}, \mathrm{König} \mathrm{A}$, Amieva $\mathrm{H}$, et al. Recommendations for the use of serious games in people with Alzheimer's disease, related disorders and frailty. Front Aging Neurosci 2014; 6:54.

22. Manera V, Ben-Sadoun G, Aalbers $T$, et al. Recommendations for the use of serious games in neurodegenerative disorders: 2016 Delphi Panel. Front Psychol 2017; 8:1243.

23. Sokolov AA, Grivaz $P$, Bove R. Cognitive deficits in multiple sclerosis: recent advances in treatment and neurorehabilitation. Curr Treat Options Neurol 2018; 20:53.

24. deBettencourt MT, Cohen JD, Lee RF, et al. Closed-loop training of attention with real-time brain imaging. Nat Neurosci 2015; 18:470-475.

25. Anguera JA, Boccanfuso J, Rintoul JL, et al. Video game training enhances cognitive control in older adults. Nature 2013; 501:97-101.

26. Ben-Sadoun $G$, Sacco $G$, Manera $V$, et al. Physical and cognitive stimulation using an exergame in subjects with normal aging, mild and moderate cognitive impairment. J Alzheimers Dis 2016; 53:1299-1314.

27. Kelly ME, Loughrey D, Lawlor BA, et al. The impact of exercise on the cognitive functioning of healthy older adults: a systematic review and meta-analysis. Ageing Res Rev 2014; 16:12-31.

28. Saez de Asteasu ML, Martinez-Velilla N, Zambom-Ferraresi F, et al. Role of physical exercise on cognitive function in healthy older adults: a systematic review of randomized clinical trials. Ageing Res Rev 2017; 37:117-134.

29. Kooiman BJ, Sheehan DP. Interacting with the past, present, and future of exergames: at the beginning of a new life cycle of video games? Society Leisure $2015 ; 38: 55-73$.

30. Stojan R, Voelcker-Rehage C. A systematic review on the cognitive benefits and neurophysiological correlates of exergaming in healthy older adults. J Clin Med 2019; 8:pii: E734.

31. Stanmore $E$, Stubbs B, Vancampfort $D$, et al. The effect of active video games on cognitive functioning in clinical and nonclinical populations: A metaanalysis of randomized controlled trials. Neurosci Biobehav Rev 2017; 78:34-43

32. Sala G, Tatlidil KS, Gobet F. Still no evidence that exergames improve cognitive ability: a commentary on Stanmore et al. Neurosci Biobehav Rev 2019; doi: 10.1016/j.neubiorev.2019.11.015. [Epub ahead of print]

33. Park JH, Park JH. Does cognition-specific computer training have better

- clinical outcomes than nonspecific computer training? A single-blind, randomized controlled trial. Clin Rehabil 2018; 32:213-222.

The authors report more health-related quality of life benefits for nonspecific video game interventions as opposed to specific computerized cognitive training.

34. Lauenroth $A$, loannidis $A E$, Teichmann $B$. Influence of combined physical and cognitive training on cognition: a systematic review. BMC Geriatr 2016; $16: 141$.

35. Sacco G, Caillaud C, Ben Sadoun G, et al. Exercise plus cognitive performance over and above exercise alone in subjects with mild cognitive impairment. J Alzheimers Dis 2016; 50:19-25.

36. Amjad I, Toor H, Niazi IK, et al. Xbox 360 Kinect Cognitive Games Improve

- Slowness, complexity of EEG, and cognitive functions in subjects with mild cognitive impairment: a randomized control trial. Games Health J 2019; 8:144-152.

Promising evidence on improvement of global cognition measures and cognitive flexibility in $\mathrm{MCl}$ through exergaming coupled with cognitive training.

37. Foroughi CK, Monfort SS, Paczynski M, et al. Placebo effects in cognitive training. Proc Natl Acad Sci U S A 2016; 113:7470-7474.

38. Anderson-Hanley C, Barcelos NM, Zimmerman EA, et al. The Aerobic and Cognitive Exercise Study (ACES) for community-dwelling older adults with or at-risk for mild cognitive impairment $(\mathrm{MCl})$ : neuropsychological, neurobiological and neuroimaging outcomes of a randomized clinical trial. Front Aging Neurosci 2018; 10:76.

39. Barcelos N, Shah N, Cohen K, et al. Aerobic and Cognitive Exercise (ACE) pilot study for older adults: executive function improves with cognitive challenge while exergaming. J Int Neuropsychol Soc 2015; 21:768-779.

40. Anderson-Hanley C, Stark J, Wall KM, et al. The interactive Physical and Cognitive Exercise System (iPACES): effects of a 3-month in-home pilot clinical trial for mild cognitive impairment and caregivers. Clin Interv Aging 2018; 13:1565-1577.

41. Mrakic-Sposta S, Di Santo SG, Franchini F, et al. Effects of combined physical and cognitive virtual reality-based training on cognitive impairment and oxidative stress in MCl patients: a pilot study. Front Aging Neurosci 2018; 10:282.
42. Cipresso P, Giglioli IAC, Raya MA, Riva G. The past, present, and future of virtual and augmented reality research: a network and cluster analysis of the literature. Front Psychol 2018; 9:2086.

43. Montana Jl, Tuena $\mathrm{C}$, Serino $\mathrm{S}$, et al. Neurorehabilitation of spatial memory using virtual environments: a systematic review. J Clin Med 2019; 8:; pii: E1516.

44. Perez-Marcos D, Bieler-Aeschlimann M, Serino A. Virtual reality as a vehicle to empower motor-cognitive neurorehabilitation. Front Psychol 2018; 9:2120.

45. Gamito $P$, Oliveira J, Morais $D$, et al. Cognitive stimulation of elderly individuals with instrumental virtual reality-based activities of daily life: pre-post treatment study. Cyberpsychol Behav Soc Netw 2019; 22:69-75.

46. Liao $Y Y$, Chen IH, Lin YJ, et al. Effects of virtual reality-based physical and

- cognitive training on executive function and dual-task gait performance in older adults with mild cognitive impairment: a randomized control trial. Front Aging Neurosci 2019; 11:162.

One of the first immersive virtual reality cognitive intervention studies showing that real-life and immersive virtual reality everyday activity training yield largely similar benefits in individuals with mild cognition.

47. La Corte V, Sperduti M, Abichou K, Piolino P. Episodic memory assessment and remediation in normal and pathological aging using virtual reality: a mini review. Front Psychol 2019; 10:173.

48. Lecouvey G, Morand A, Gonneaud J, et al. An impairment of prospective memory in mild Alzheimer's disease: a ride in a virtual town. Front Psychol $2019 ; 10: 241$.

49. Tuena $C$, Serino $S$, Dutriaux L, et al. Virtual enactment effect on memory in young and aged populations: a systematic review. J Clin Med 2019; 8:; pii: E620.

50. Manera V, Chapoulie E, Bourgeois J, et al. A feasibility study with image-based rendered virtual reality in patients with mild cognitive impairment and dementia. PLoS One 2016; 11:e0151487.

51. Livingston G, Sommerlad A, Orgeta V, et al. Dementia prevention, intervention, and care. Lancet 2017; 390:2673-2734.

52. Ngandu T, Lehtisalo J, Solomon A, et al. A 2 year multidomain intervention of diet, exercise, cognitive training, and vascular risk monitoring versus control to prevent cognitive decline in at-risk elderly people (FINGER): a randomised controlled trial. Lancet 2015; 385:2255-2263.

53. Belleville $S$, Moussard $A$, Ansaldo Al, et al. Rationale and protocol of the ENGAGE study: a double-blind randomized controlled preference trial using a comprehensive cohort design to measure the effect of a cognitive and leisurebased intervention in older adults with a memory complaint. Trials 2019; 20:282.

54. Jirayucharoensak $S$, Israsena $P, P a n-N g u m ~ S$, et al. A game-based neurofeed-

- back training system to enhance cognitive performance in healthy elderly subjects and in patients with amnestic mild cognitive impairment. Clin Interv Aging 2019; 14:347-360.

An interesting study comparing neurofeedback to cognitive exergame training.

55. Liu $Y$, Tan W, Chao C, et al. A review of the application of virtual reality technology in the diagnosis and treatment of cognitive impairment. Front Aging Neurosci 2019; 11:280.

56. Ouellet E, Boller B, Corriveau-Lecavalier N, et al. The Virtual Shop: a new immersive virtual reality environment and scenario for the assessment of everyday memory. J Neurosci Methods 2018; 303:126-135.

57. Parsons TD, Barnett M. Validity of a newly developed measure of memory: feasibility study of the virtual environment grocery store. J Alzheimers Dis 2017; 59:1227-1235.

58. Buitenweg JIV, Van De Ven RM, Ridderinkhof KR, Murre JMJ. Does cognitive flexibility training enhance subjective mental functioning in healthy older adults? Neuropsychol Dev Cogn B Aging Neuropsychol Cogn 2019; 26:688-710.

59. Flak MM, Hol HR, Hernes SS, et al. Adaptive computerized working memory training in patients with mild cognitive impairment. A randomized double-blind active controlled trial. Front Psychol 2019; 10:807.

60. Rolle CE, Anguera JA, Skinner SN, et al. Enhancing spatial attention and working memory in younger and older adults. J Cogn Neurosci 2017; 29:1483-1497.

61. Ballesteros S, Voelcker-Rehage C, Bherer L. Editorial: cognitive and brain plasticity induced by physical exercise, cognitive training, video games, and combined interventions. Front Hum Neurosci 2018; 12:169.

62. Mattioli F, Stampatori C, Bellomi F, et al. A RCT comparing specific intensive cognitive training to aspecific psychological intervention in RRMS: the SMICT Study. Front Neurol 2014; 5:278.

63. Sumowski JF, Chiaravalloti N, Deluca J. Retrieval practice improves memory in multiple sclerosis: clinical application of the testing effect. Neuropsychology 2010; 24:267-272.

64. Whitlock LA, McLaughlin AC, Allaire JC. Individual differences in response to cognitive training: using a multimodal, attentionally demanding game-based intervention for older adults. Comput Hum Behav 2012; 28:1091-1096.

65. Peter J, Schumacher LV, Landerer V, et al. Biological factors contributing to the response to cognitive training in mild cognitive impairment. J Alzheimers Dis 2018; 61:333-345.

66. Sokolov AA, Zeidman $\mathrm{P}$, Erb $\mathrm{M}$, et al. Structural and effective brain connectivity underlying biological motion detection. Proc Natl Acad Sci U S A 2018; 115:E12034-E12042. 